\title{
Anticardiolipin antibodies in patients with multiple sclerosis do not represent a subgroup of patients according to clinical, familial, and biological characteristics
}

\author{
O Heinzlef, B Weill, C Johanet, V Sazdovitch, S Caillat-Zucman, E Tournier-Lasserve, \\ E Roullet
}

Background: In multiple sclerosis (MS), case control studies have shown that anticardiolipin antibodies ( $a C L A b)$ are more frequent than in the general population and that $\mathrm{aCL} \mathrm{Ab}$ positivity may be associated with specific clinical characteristics.

Objectives: To determine whether patients with MS who are positive for $a C L A b$ have specific characteristics.

Methods: 285 consecutive patients with MS were tested for $\mathrm{aCL} \mathrm{Ab}$ positivity. Patients also underwent complete autoimmune screening and were systematically evaluated for clinical characteristics and individual or family history of autoimmune disease.

Results: aCL Ab positivity was found in 42 patients (15\%). The main isotype was aCL lgM (32 patients, $11 \%$ ). Demographics and clinical characteristics including sex, age at onset, course of the disease, expanded disability status scale score, and progression index were not different between $a C L A b$ positive and $a C L A b$ negative patients. Clinical systems involved at onset or during the course of the disease were not different from what is usually observed in MS. aCL Ab positivity was not associated with an increased frequency of autoimmune disease and was not predictive of a family history of autoimmune disease. Patients positive for aCL IgM were more frequently positive for the presence of non-organ specific antibodies $153 \% \mathrm{v}$ $39 \%$, respectively, $p=0.02$ ).

Conclusions: These results do not support the hypothesis that patients with MS with aCL Ab constitute a subgroup of MS according to demographic clinical and familial characteristics. The greater frequency of other antibodies in $a C L A b$ positive patients suggests that they only reflect a more general autoimmune activation in MS

$\mathrm{T}$ here is evidence in multiple sclerosis (MS) for heterogeneity in the course of the disease, site of involvement, prognosis, and response to treatment. Previous studies have shown that patients with MS more often have immunological abnormalities than those without. ${ }^{12}$ Whether patients with MS who have associated autoimmune disease or biological markers of extraneurological autoimmunity have a different phenotype is still a matter of debate. ${ }^{3}$ Antiphospholipids are considered to be one of the most commonly identified acquired haematological risk factors for thrombo-occlusive events. ${ }^{4}$ Other neurological diseases such as epilepsy, idiopathic intracranial hypertension, migraine, and chorea have been associated with antiphospholipid antibodies. ${ }^{5-7}$

There have been some case reports or series linking anticardiolipin antibodies (aCL Ab) and MS, optic neuritis, or trans- verse myelopathy. ${ }^{8-15}$ Neuroradiological studies have shown an association between high intensity white matter lesions and the presence of $\mathrm{aCL} \mathrm{Ab}$, and some authors have suggested that $\mathrm{aCL} \mathrm{Ab}$ are implicated in the pathogenesis of MS. However, others did not confirm that patients with MS and antiphospholipid antibodies constitute a subgroup of patients, which suggests that aCL Ab may be a non-specific marker of central nervous system injury. ${ }^{16}{ }^{17}$.

To investigate further the relation between aCL Ab and MS, we determine aCL IgG and IgM titres in a cohort of patients with MS. We then compared the clinical characteristics of patients according to their aCL Ab status. As familial aggregation of $\mathrm{aCL} \mathrm{Ab}$ and an association between $\mathrm{aCL} \mathrm{Ab}$ and systemic lupus erythematosus (SLE) or SLE like syndrome have been reported, we also examined the relation between $\mathrm{aCL} \mathrm{Ab}$ positivity in patients with MS and the presence of autoimmune diseases in relatives.

\section{PATIENTS AND METHODS \\ Patients}

Patients were consecutive patients examined as in- or outpatients at our clinic ${ }^{18}$ during a 12 month period starting 1 December 1992 who had a clinically or laboratory confirmed diagnosis of definite MS. ${ }^{19}$ The diagnosis was checked with particular attention to patients with antinuclear antibodies and MS; none of them fulfilled criteria for SLE, primary antiphospholipid syndrome, or any connective tissue disease.

\section{Family medical history}

Medical records were reviewed and a detailed family medical history was taken in person or by telephone interview with the index case patient. Diagnoses of MS and autoimmune diseases in patients and family members were documented by review of medical records. Present age, age at onset, MS course, ${ }^{20}$ duration of MS, treatment, and expanded disability status scale (EDSS) score ${ }^{21}$ were recorded. Signs at onset and patients were classified according to the functional system involved. Magnetic resonance imaging and cerebrospinal fluid data were not available for all patients and are not reported here. This study was approved by the ethics committee of our hospital and patients gave informed consent.

Of the 297 patients enrolled in the study, eight (2.7\%) could not be interviewed about their family history. Four patients

Abbreviations: $a C L A b$, anticardiolipin antibody; ATM, acute transverse myelopathy; EDSS, expanded disability status scale; GPL, antibody reactivity of $1 \mathrm{mg} / \mathrm{ml}$ of purified anticardiolipin $\lg G ; M P L$, antibody reactivity of $1 \mathrm{mg} / \mathrm{ml}$ of purified anticardiolipin lgM; MS, multiple sclerosis; SLE, systemic lupus erythematosus 
Table 1 Demographics and clinical characteristics of patients according to anticardiolipin (aCL) antibody positivity

\begin{tabular}{|c|c|c|c|c|}
\hline & \multicolumn{2}{|l|}{$a C L \lg G$} & \multicolumn{2}{|l|}{$a C L \lg M$} \\
\hline & Positive ( $n=12$ ) & Negative $(n=273)$ & Positive $(n=32)$ & Negative $(n=257)$ \\
\hline Men & $1(8 \%)$ & $85(31 \%)$ & $11(35 \%)$ & $76(30 \%)$ \\
\hline Women & $11(92 \%)$ & $188(69 \%)$ & $21(65 \%)$ & $181(70 \%)$ \\
\hline Age (years) & 44 (12) & 44 (11) & 48 (9) & $43(11)$ \\
\hline Age at onset (years) & $30(10)$ & $30(9)$ & 34 (10) & $29(9)$ \\
\hline EDSS & $4(1.7)$ & $4.3(2.2)$ & $4.9(1.34)$ & $4.3(2.2)$ \\
\hline Progression rate* & $0.4(0.3)$ & $0.4(0.4)$ & $0.5(0.4)$ & $0.4(0.4)$ \\
\hline \multicolumn{5}{|l|}{ Course of the disease } \\
\hline RR & $7(59 \%)$ & $159(58 \%)$ & $13(41 \%)$ & $155(60 \%)$ \\
\hline SP & $3(25 \%)$ & $68(25 \%)$ & $10(31 \%)$ & $62(24 \%)$ \\
\hline PP & $2(16 \%)$ & $32(12.5 \%)$ & $7(22 \%)$ & 27 (11\%) \\
\hline Undetermined & $0(0 \%)$ & $14(4.5 \%)$ & $2(6 \%)$ & $13(5 \%)$ \\
\hline
\end{tabular}

were not tested for aCL Ab. The study population consisted of 285 patients ( 199 women, 86 men) ranging in age from 20 to 68 years (mean (SD) 44 (11) years).

\section{Antiphospholipid antibody assay}

$\mathrm{aCL} \mathrm{Ab}$ were sought using the enzyme linked immunoassay described by Harris et al. ${ }^{22}$ GPL and MPL units were defined as the antibody reactivity of $1 \mathrm{mg} / \mathrm{ml}$ of purified aCL IgG or IgM, respectively. The test is considered positive when aCL IgG are $>15$ U GPL or when aCL IgM are > 6 U MPL.

\section{RESULTS}

Twelve patients (4\%) were positive for aCL IgG, 32 (11\%) for aCL IgM, and $42(15 \%)$ for aCL IgG or IgM. aCL Ab titres were usually low, as only one patient had aCL IgG $\geqslant 40$ GPL units and three had aCL IgM $\geqslant 40$ GPL units.

\section{Correlations with MS characteristics}

Patients positive for aCL IgM were older than those who were negative. Age at onset and sex ratio were similar in the positive and negative groups. There was no difference between the groups with respect to EDSS scores, course of the disease, and progression rates (table 1). Functional systemic involvement could be determined in only 36 of 42 patients. Whatever the aCL isotype, the sensory system was the most frequently involved at onset (56\%) followed by motor system $(50 \%)$, brainstem (28\%), optic neuritis (14\%), and cerebellum, bladder, and bowel (5\% each). During the course of the disease, the motor and sensory systems and the brainstem were the most frequently involved $(86 \%, 81 \%$, and $78 \%$, respectively), followed by cerebellum $(70 \%)$, optic neuritis $(43 \%)$, and bladder and bowel (45\%). One patient had epilepsy, three had cognitive impairment, and three had paroxystic cephalalgia (one migraine). One woman had a miscarriage, but none had phlebitis, pulmonary embolism, or cerebral infarction.

\section{Frequency of autoimmune abnormalities}

aCL positivity was not associated with an increased frequency of autoimmune diseases. None of our patients had SLE. Only one patient positive for aCL IgG and two positive for aCL IgM had another autoimmune disease. The presence of aCL IgG or IgM was not predictive of a family history of autoimmune disease. Patients positive for aCL IgM were more frequently positive for another antibody $(60 \% \vee 38 \%$, respectively, $\mathrm{p}=0.02)$. ACL IgM positivity was also associated with the presence of non-organ specific antibodies (53\% v 39\%, respectively, $\mathrm{p}=0.02$ ).

\section{DISCUSSION}

This study is a negative one, as we did not find any association between the presence of aCL Ab and the clinical characteristics of MS. We have a large cohort of consecutive patients who were not selected for arbitrary characteristics and we avoided a recruitment bias.

Our study was limited in power by the low frequency of patients positive for aCL Ab, not different from the general population. The power of the study to detect a twofold effect (such as doubling the proportion of primary progressive patients with aCL IgM) was about $50 \%$. However, a positive result with IgM but not with IgG would probably be of limited significance, as aCL IgM positivity has less specificity and may be present after infection or the administration of drugs. To exclude false positivity it is useful to analyse serially patients with positive samples but this was not done in our study.

In patients with MS, some clinical characteristics have been associated with aCL Ab positivity. In Japanese patients with definite MS, Fukazawa et al ${ }^{12}$ found that 2 of 38 patients (5.3\%) were positive for aCL Ab. Ten of their patients had an acute transverse myelopathy (ATM) (26\%) and both patients who were positive for aCL Ab had recurrent ATM and optic neuritis. We did not observe ATM, and the frequency of optic neuritis at onset or during the course of the disease was comparable with published frequencies. This difference may be explained by the high frequency of ATM in Japanese patients and the small size of their sample. In a more recent study involving 322 patients referred to a rheumatology clinic and evaluated for antiphospholipid antibody positivity, 26 (8\%) had a diagnosis of MS. Clinically, seven (25\%) had ATM and eight (32\%) had optic neuritis. However, patients were only tested for aCL Ab positivity if they had antiphospholipid related symptoms or a family history of antiphospholipid related disease, which may account for the unusual frequency of ATM in this subgroup of patients. ${ }^{14}$

More recently, Karussis et a l $^{15}$ found a high frequency of aCL Ab (27\%) in 100 patients with MS selected for "unusual"clinical characteristics (headaches, slowly progressive myelopathy, and absence of oligoclonal bands in the cerebrospinal fluid). They found a high frequency of a progressive course with predominantly cerebellar syndrome and myelopathy. They also suggested that the progression rate was slower than that expected for classic MS. ${ }^{15}$ We used a different approach and our results do not support the hypothesis that patients with $\mathrm{aCL} \mathrm{Ab}$ have particular clinical characteristics according to the course of the disease, EDSS, or progression index. Moreover, the frequency of systemic impairment was comparable between the positive and negative groups. Only three patients had paroxysmal headache $(7 \%)$ and none had chronic 
headaches. Autoimmune diseases and family history for autoimmune diseases were not more frequent in patients with MS with aCL Ab. As our patients were consecutively included in the study regardless of their "usual" or "unusual" clinical characteristics, methodological considerations may account for these differences. However, it is interesting that aCL Ab titres were higher in the study of Karussis et al ${ }^{15}$; another explanation may be that in selecting only patients with definite MS, we excluded some frontier patients with atypical MS and disease associated with aCL Ab positivity.

Our results are in keeping with those of Tourbah et al, ${ }_{1}^{16}$ who followed up 64 patients with MS and autoimmune abnormalities (including aCL Ab) for four to five years. They did not find significant differences between patients with and those without autoimmune abnormalities, and the final diagnosis was MS in 50 patients with unusual biological findings (aCL Ab in 8 of 50). ${ }^{16}$ This was confirmed by Sastre-Garriga et al,${ }^{17}$ who did not find any specific clinical characteristics in six patients with MS and positive for aCL Ab among 296 patients tested. Finally, the association between aCL Ab positivity and any other antibodies supports the hypothesis that aCL Ab in MS only reflects a more general autoimmune activation.

Therefore, it is not appropriate to test patients with MS systematically for aCL Ab except on a case by case basis.

\section{ACKNOWLEDGEMENT}

Purified aCL IgG and IgM were kindly provided by EN Harris from the Department of Medicine, Morehouse School of Medicine, Atlanta, Georgia, USA.

\section{Authors' affiliations}

O Heinzlef, V Sazdovitch, E Roullet, Department of Neurology, Tenon Hospital, 4 rue de la Chine, 75020 Paris, France

C Johanet, Department of Immunology, Saint-Antoine Hospital, Paris, France

B Weill, Department of Biological Haematology, Cochin Hospital, Paris, France

S Caillat-Zucman, Department of Biological Immunology, Necker Hospital, Paris, France

E Tournier-Lasserve, INSERM EPI 99-21, Lariboisière Medical Faculty, Paris, France

Correspondence to: Dr O Heinzlef, Service de Neurologie de l'Hôpital Tenon, 4 rue de la Chine, 75020 Paris, France;

olivier.heinzlef@tnn.ap-hop-paris.fr

Received 11 June 2001

In revised form 14 December 2001

Accepted 15 January 2002

\section{REFERENCES}

1 De Keyser J. Autoimmunity in multiple sclerosis. Neurology 1988;38:371-4
2 Seyfert S, Klapps P, Meisel C, et al. Multiple sclerosis and other immunologic diseases. Acta Neurol Scand 1990;81:37-42

3 Collard R, Koehler R, Mattson D. Frequency and significance of antinuclear antibodies in multiple sclerosis. Neurology 1997;49:857-61

4 Alarcon-Segovia D, Deleze M, Oria CV, et al. Antiphospholipid antibodies and the antiphospholipid syndrome in systemic lupus erythematosus: a prospective analysis of 500 consecutive patients. Medicine (Baltimore) 1989;68:353-65.

5 Levine SR, Welch KMA. The spectrum of neurological disease associated with antiphospholipid antibodies: lupus anticoagulants and anticardiolipin antibodies. Arch Neurol 1987;44:876-83.

6 Leker RR, Steiner I. Anticardiolipin antibodies are frequently present in patients with idiopathic intracranial hypertension. Arch Neurol 1998:55:817-20.

7 Briley DP, Coull BM, Goodnight SH. Neurological disease associated with antiphospholipid antibodies. Ann Neurol 1989;25:221-7.

8 Campi A, Filippi $M$, Comi G, et al. Recurrent acute transverse myelopathy associated with anticardiolipin antibodies. Am J Neuroradiol 1998;19:781-6.

9 Medina-Rodriguez F, Garcia E, Fraga A. Transverse myelitis and antiphospholipid antibodies. J Rheumatol 1990;17:9.

10 Lavalle C, Pizarro S, Drenkard C, et al. Transverse myelitis: a manifestation of systemic lupus erythematosus strongly associated with antiphospholipid antibodies. J Rheumatol 1990;17:34-7.

11 Gerber SL, Cantor LB. Progressive optic atrophy and the primary antiphospholipid antibody syndrome. Am J Ophthalmol 1990;110:443-4.

12 Fukazawa T, Moriwaka F, Mukai M, et al. Anticardiolipin antibodies in Japanese patients with multiple sclerosis. Acta Neurol Scand 1993;88:184-9.

13 Sugiyama Y, Yamamoto T. Characterization of serum anti-phospholipid antibodies in patients with multiple sclerosis. Tohoku J Exp Med 1996; 178:203-15.

14 lido JW, Conti-Kelly AM, Greco P, et al. Anti-phospholipid antibodies in patients with multiple sclerosis and MS-like illnesses: MS or APS? Lupus 1999;8: 109-15.

15 Karussis D, Leker R, Ashkenazi A, et al. A subgroup of multiple sclerosis patients with anticardiolipin antibodies and unusual clinical manifestations: do they represent a new nososlogical entity. Ann Neurol 1998;44:629-34.

16 Tourbah A, Clapin A, Gout O, et al. Systemic autoimmune features and multiple sclerosis: a 5-year follow-up study. Arch Neurol 1998;55:517-21

17 Sastre-Garriga J, Reverter JC, Font J, et al. Anticardiolipin antibodies are not a useful screening tool in a nonselected large group of patients with multiple sclerosis. Ann Neurol 2001;49:408-11.

18 Roullet E, Verdier-Taillefer M, Amarenco P, et al. Pregnancy and multiple sclerosis: longitudinal follow-up of 125 remittent patients. J Neurol Neurosurg Psychiatry 1993;56:1062-5

19 Poser CM, Paty DW, Scheinberg L, et al. New diagnostic criteria for multiple sclerosis: guidelines for research protocols. Ann Neurol 1983;13:227-31.

20 Lublin FD, Reingold S. Defining the clinical course of multiple sclerosis: results of an international survey. Neurology 1996;46:907-11.

21 Kurtzke J. Rating neurologic impairment in multiple sclerosis: an expanded disability status scale (EDSS). Neurology 1983;33:1444-52.

22 Harris EN, Gharavi AE, Hughes GRV. Use of an enzyme-linked immunosorbent assay and of inhibition studies to distinguish between antibodies to cardiolipin from patients with syphilis or autoimmune disorders. J Infect Dis 1988;157:23-32. 